\title{
A Pilot Study of Intervention With a Mobile Application Visualizing the Macronutrient Content for Type 2 Diabetes at a Japanese Center
}

\author{
Asako Tsunemi ${ }^{\mathrm{a}}$, Junko Sato ${ }^{\mathrm{a}, \mathrm{e}} \mathbb{\oplus}$, Sakae Sugimoto ${ }^{\mathrm{b}}$, Yuko Iwagaki ${ }^{\mathrm{b}}$, Mari Enomoto ${ }^{\mathrm{b}}$, Yuki Someya ${ }^{\mathrm{a}}$, \\ Mai Kiya ${ }^{a}$, Eiko Matsuhashia ${ }^{a}$ Yuka Wakabayashia ${ }^{a}$, Takashi Funayama ${ }^{\text {a }}$,Tomoya Mita ${ }^{\text {, }}$ \\ Toyoyoshi Uchida ${ }^{a}$, Takeshi Miyatsuka ${ }^{a}$, Kosuke Azuma ${ }^{a}$, Tomoaki Shimizu ${ }^{a}$, \\ Akio Kanazawa ${ }^{\text {a }}$, Hiroaki Satoh ${ }^{\mathrm{a}}$, Hirotaka Watada ${ }^{\mathrm{a}, \mathrm{c}, \mathrm{d}}$
}

\begin{abstract}
Background: Estimating the nutritional content of food is essential for self-management in people with type 2 diabetes mellitus, but it is a difficult skill to learn. The aim of this study was to investigate how diabetes management was impacted by the ability of patients to search for items they ate from a database of 26,300 different foods, and to visualize nutritional intake using the Japanese mobile application (app) "Calomeal."

Methods: This was a single-arm, single-center, pilot study. Eighteen outpatients with type 2 diabetes mellitus used the "Calomeal" app for 3 months. The primary endpoint was change in hemoglobin A1c $(\mathrm{HbA} 1 \mathrm{c})$. Secondary endpoints were changes in body weight $(\mathrm{BW})$, lipid parameters, and quality of life scores.

Results: The baseline characteristics of the study subjects were as follows: age: $53.4 \pm 7.8$ years; male/female ratio: 11/7; HbA1c: 7.9 (7.58 - 8.23)\%; and body mass index (BMI): 25.17 (21.63 - 28.59) $\mathrm{kg} / \mathrm{m}^{2}$. Significant reductions in $\mathrm{HbAlc}$ and BMI were observed over 3 months (HbA1c: $7.9(7.58-8.23) \%$ to $7.6(7.3-8.23) \%, P=$ 0.0410 ; BMI: 25.17 (21.63 - 28.59) to $24.54(21.57$ - 27.81) kg/m², $\mathrm{P}=0.0057)$. Reductions in HbAlc and BMI both correlated with decreased carbohydrate intake estimated by the mobile app.
\end{abstract}

Conclusions: Japanese patients who used their smartphones to visualize their nutritional intake using the "Calomeal" app demonstrated

Manuscript submitted July 9, 2021, accepted July 23, 2021

Published online August 30, 2021

aDepartment of Metabolism and Endocrinology, Juntendo University Graduate School of Medicine, Tokyo, Japan

${ }^{b}$ Department of Nutrition, Juntendo University Hospital, Tokyo, Japan

${ }^{\mathrm{c}}$ Center for Therapeutic Innovations in Diabetes, Juntendo University Graduate School of Medicine, Tokyo, Japan

${ }^{\mathrm{d}}$ Center for Identification of Diabetic Therapeutic Targets, Juntendo University Graduate School of Medicine, Tokyo, Japan

${ }^{e}$ Corresponding Author: Junko Sato, Department of Metabolism and Endocrinology, Juntendo University Graduate School of Medicine, 2-1-1 Hongo, Bunkyo-ku, Tokyo 113-8421, Japan. Email: jsato@juntendo.ac.jp

doi: https://doi.org/10.14740/jocmr4558 improved short-term glycemic control and BMI. Although the validity of the results should be tested in future randomized controlled trials, this approach may be a clinical option for improving self-management in Japanese patients with type 2 diabetes mellitus.

Keywords: Medical nutrition therapy; Mobile applications; Selfmanagement; Type 2 diabetes mellitus

\section{Introduction}

According to the National Health and Nutrition survey of Japan, $12.1 \%$ of the Japanese population was estimated to have diabetes in 2016 [1]. The majority of them had type 2 diabetes mellitus, and the prevalence of diabetes in Japan has been increasing every year [2]. To improve prognosis and quality of life (QOL) in type 2 diabetes mellitus, it is important to manage the risk factors for macro- and microvascular disease. The fundamental way to achieve this is through self-management of diabetes mellitus, a core component of which is medical nutrition therapy. Therefore, nutritional guidance by registered dietitians is an effective method, as described in many guidelines worldwide [3-5]. However, it is difficult for patients to know what and how much they can eat in daily life. In addition, dietary habits vary depending on culture, religion, economic state, and individual preferences. In particular, since Japanese cuisine involves many ingredients, it is difficult for Japanese to monitor their nutritional intake.

Many types of healthcare-related mobile applications (apps) have been developed globally, some of which have been shown by systematic reviews to be effective for glycemic control $[6,7]$. However, most currently available and evaluated diabetes-related apps are not available in languages other than English [8], yet. Accordingly, healthcare apps are not yet widely used as a formal treatment tool in Asian countries, including Japan. The Japanese app "Calomeal" that the patients are able to easily download on their smartphones with basically costfree, enables them to search a database for the foods they eat or drink, and to visualize their nutritional content. The aim of 
this pilot study was to investigate the efficacy of intervention using a mobile app on the management of Japanese patients with diabetes mellitus.

\section{Materials and Methods}

\section{Design}

This was a single-center, single-arm, open-label study. The study protocol was approved by the Ethics Committee of Juntendo University Hospital (Approval No.18-146) on November 26, 2018. The study conformed to the provisions of the Declaration of Helsinki and each participant provided written informed consent.

\section{Study subjects}

The study was conducted from December 2018 to December 2019. The inclusion criteria were the following: 1) type 2 diabetes mellitus; 2) adults aged from 20 to 65 years; and 3) hemoglobin A1c (HbA1c) values from $7.5 \%$ to $9 \%$. The exclusion criteria were as follows: 1) type 1 diabetes; 2) steroid-induced diabetes; 3) currently pregnant; 4) stage 3 - 5 nephropathy; 5) current cancer diagnosis; and 6) past experience using similar apps. Among 32 patients at Juntendo University Hospital who met the inclusion criteria, agreement to participate was obtained from 22 patients, two of whom were excluded because they did not use smartphones. Thus, we recruited 20 patients with type 2 diabetes mellitus.

\section{Healthcare app "Calomeal"}

"Calomeal", one of commercially available Japanese mobile apps, was initially developed by Life Log Technology, Inc., in 2016. It has several functions, including artificial intelligencebased image analysis and identification, manual search for numerous foods and subsequent analysis, calculation of exercise-induced energy consumption, and logging of body weight (BW) and body fat percentage. This study used only two functions, specifically manual search and analysis of foods and BW logging, which were available without payment to the company. The app enabled patients to search for what they ate or drank from a database of 26,300 different foods and products, and to visualize nutritional content on their smartphones. The database covered not only everyday home-cooked items, but also foods available in many popular Japanese restaurants and convenience stores. A graph of daily energy intake and the intake of different nutritional components was displayed on the smartphone screen, and if the target intake for any of these was exceeded, the line turned red (Fig. 1).

In this study, we set the target daily energy intake at 30 kcal per target BW (body height $\left.(\mathrm{m})^{2} \times 22\right)(\mathrm{kg})$, and aimed for carbohydrates, proteins, and lipids to comprise $50 \%, 20 \%$, and $30 \%$ of the daily energy intake, respectively, based on the Japanese Clinical Practice Guidelines published by the Japan
Diabetes Society in 2018 [9]. Patients recorded the details of each meal they ate. If at any point they did not eat a meal, they could select the "skipped meal" option displayed on the screen, which enabled us to distinguish the lack of information from data loss. Patients were considered to be adherent to their use of the app if they recorded the number of meals eaten per day on at least 3 days per week ( 2 weekdays and 1 weekend day). They could use their smartphones to visualize not only daily data but also weekly or monthly graphs.

\section{Observation variables and schedules}

The 20 recruited patients with type 2 diabetes mellitus used the "Calomeal" app for 3 months while undergoing regular treatment. Throughout the study period, they did not receive any advice about nutrition therapy from either staff members or the registered dietician.

At the beginning and end of the study, information regarding 3 days' meals was used to compare nutrient intake estimated by the app and the registered dietitian. Considering the difficulty in estimating nutrient intake, we asked patients to estimate energy intake only.

The 3-day record of weighed/measured was assessed by the registered dietitian based on photos of meals provided by each patient in a face-to-face meeting. The total energy and nutrient intake were calculated using the Super Nutrition Calculation System, Healthy Maker Pro 501 Series (Mushroomsoft, Okayama, Japan), a software program that is widely used in the nutrition departments of Japanese hospitals. Patients were required to take photos of meals from both an overhead perspective and from an angle of approximately $45^{\circ}$, and also to place a standard reference object (a $8.5 \mathrm{~cm} \times 5.0 \mathrm{~cm}$ card $)$ next to the foods to clarify the size.

Patients' BW and blood pressure were measured by the staff at the beginning and end of the study. Blood and urine samples were obtained at the same time, and serum lipids (low-density lipoprotein cholesterol (LDL-C), high-density lipoprotein cholesterol (HDL-C), triglycerides (TG)), blood glucose (BG), HbA1c, liver enzymes, serum creatinine, and urinary albumin were measured with standard techniques.

\section{Diabetes treatment satisfaction questionnaire (DTSQ) and problem areas in diabetes (PAID)}

The DTSQ and PAID were administered to evaluate patient QOL at the beginning and end of the study. The DTSQ consists of eight items scored on a scale of 0 - 6 [10]. Items $1,4,5,6,7$, and 8 are summed to calculate the treatment satisfaction score (range: 0 - 36). The higher the score is, the greater the satisfaction with treatment. In this study we excluded item 2, "perceived frequency of hyperglycemia," and item 3, "perceived frequency of hypoglycemia." The PAID scale is widely regarded as the first patient-reported outcome measure to assess diabetes distress in adults [11]. The PAID comprises a 5-point scale ranging from "not a problem" (score of 0 ) to "serious problem" (score of 4). Multiplying the summed score by 1.25 


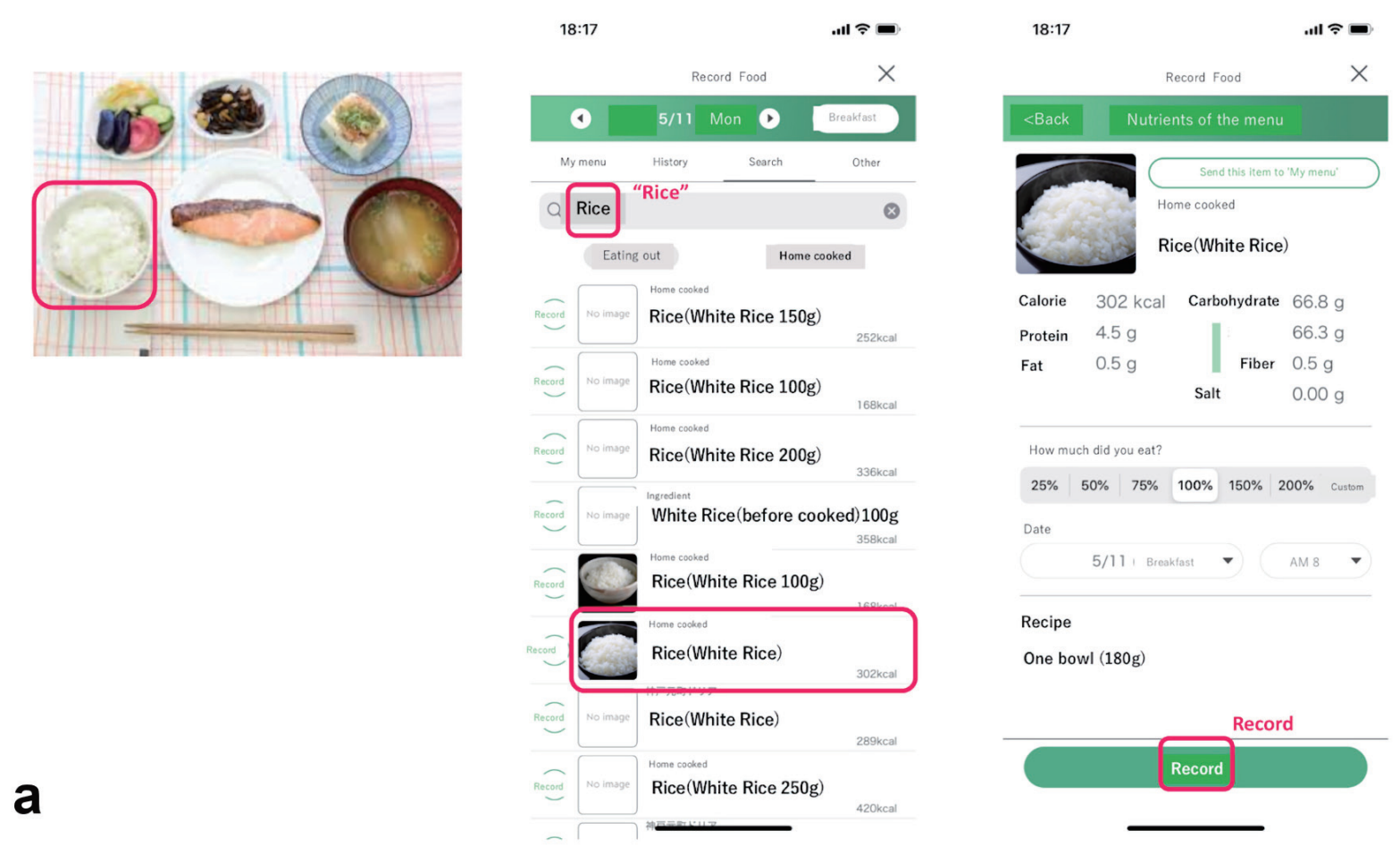

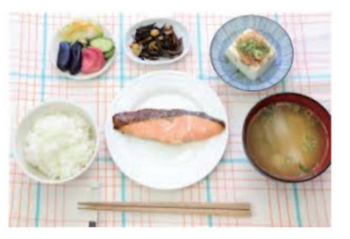
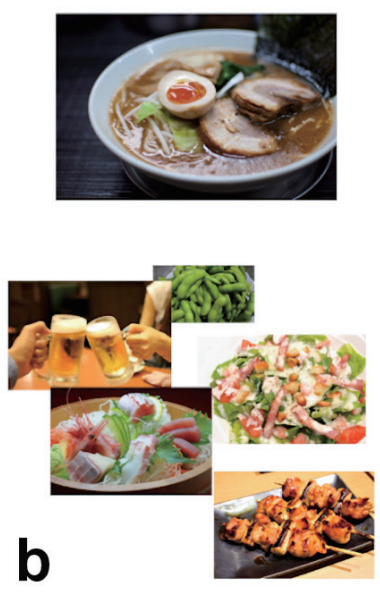
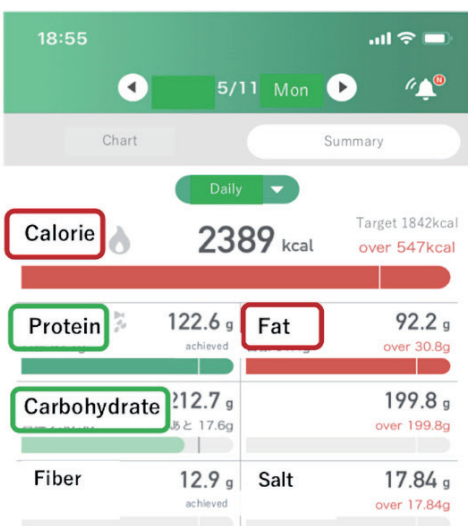

\begin{tabular}{|l}
\hline Height : $167 \mathrm{~cm}$ \\
$>$ Energy: $1842 \mathrm{kcal}(\times 30 / \mathrm{kg} \cdot \mathrm{iBW})$ \\
$>$ Pro $: 92.1 \mathrm{~g} \quad(20 \%$ energy $)$ \\
$>$ Fat $: 61.4 \mathrm{~g}(30 \%$ energy $)$ \\
$>$ Carbo $: 230.3 \mathrm{~g}(50 \%$ energy $)$ \\
\hline
\end{tabular}
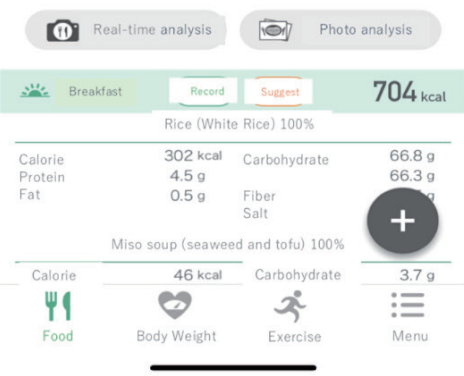

Figure 1. Sample app screenshots (translated into English). When eating one bowl of white rice, the participant searched for the word "Rice" and then selected the closest match from the presented list, including the amount they ate (a). After logging all meals of the day, the home screen displayed the total daily intake of energy and various nutrients. If the participant exceeded the recommended amount of any of these, the corresponding line would turn red (b). BW: body weight; Pro: protein; Carbo: carbohydrate; app: application. 
Table 1. Reliability and Validity of Estimates by the "Calomeal" App, Compared to the Estimates of a Registered Dietitian

\begin{tabular}{|c|c|c|c|c|c|}
\hline & \multirow{3}{*}{ ICC $(2,36)(95 \%$ CI $)$} & \multicolumn{4}{|c|}{ Bland-Altman analysis } \\
\hline & & \multirow{2}{*}{$\begin{array}{l}\text { Fixed bias } \\
95 \% \text { CI }(\%) \\
\end{array}$} & \multicolumn{3}{|c|}{ Proportional bias } \\
\hline & & & \multicolumn{2}{|c|}{ Test of no correlation } & Bias \\
\hline Protein & $0.92(0.83-0.96)$ & $-0.6-1.4$ & $\mathrm{R}=0.01$ & $\mathrm{P}=0.99$ & None \\
\hline Lipid & $0.88(0.76-0.94)$ & $-0.8-1.7$ & $\mathrm{R}=0.03$ & $\mathrm{P}=0.85$ & None \\
\hline Salt & $0.84(0.67-0.92)$ & $-3.5-2.0$ & $R=-0.19$ & $\mathrm{P}=0.26$ & None \\
\hline
\end{tabular}

An ICC over 0.81 indicates high reliability. ICC: intraclass correlation coefficient; app: application; Cl: confidence interval.

yields the overall PAID score, which can range from 0 to 100 ; higher scores indicate greater levels of distress [12].

\section{Endpoints}

The primary endpoint was change in HbAlc after 3 months. Secondary endpoints were changes in BW, lipid parameters, and QOL scores (DTSQ/PAID).

\section{Statistical analysis}

Data were expressed as means \pm standard deviation (SD) for normally distributed data and medians (interquartile range) for data with skewed distributions. The Mann-Whitney U test, Wilcoxon signed-rank test, and Spearman's rank correlation coefficient test were used for data analysis. A P value $<0.05$ denoted the presence of a statistically significant difference. Statistical analyses were conducted using the JMP statistical software package, version 10.0.2 (SAS Institute, Cary, NC, USA). The validity and reliability of nutrient estimates obtained using the "Calomeal" app were compared to those calculated by a registered dietitian by intraclass correlation coefficients (ICCs) and Bland-Altman analysis using SPSS for Windows version 17.0, (SPSS Inc., Chicago, IL, USA). ICCs were interpreted as follows: $<0$, poor agreement; $0.01-0.20$, slight agreement; 0.21 - 0.40, fair agreement; $0.41-0.60$, moderate agreement; 0.61 - 0.80, substantial agreement; and 0.81 - 1.00, almost perfect agreement [13]. The analysis excluded patients who dropped out during the study and other missing data.

\section{Results}

\section{Baseline characteristics}

During the study period, two patients were considered to have dropped out because they stopped going to the hospital, and therefore we analyzed the remaining 18 patients. The mean age of the study subjects was $53.4 \pm 7.8$ years, with 11 males and seven females. The mean duration of diabetes mellitus was
$13.7 \pm 8.2$ years, the mean $\mathrm{HbA} 1 \mathrm{c}$ was $7.9(7.58-8.23) \%$, and the mean BMI was 25.17 (21.63 - 28.59) kg/m². Regarding medications for diabetes, patients received oral hypoglycemic agents alone $(\mathrm{n}=10)$, with a glucagon-like peptide-1 (GLP-1) analogue $(n=4)$, with insulin $(n=3)$, or with a GLP-1 analogue and insulin $(n=1)$. Most participants had no diabetic microvascular complications (retinopathy: no diabetic retinopathy $(n=14)$, simple retinopathy $(n=3)$, proliferative retinopathy $(n=1)$; nephropathy: stage $1(n=17)$, stage $2(n=1))$.

\section{Reliability and validity of nutrient estimates using the "Calomeal" app}

ICCs and Bland-Altman analysis were used to compare the reliability and validity of nutrient estimates using the "Calomeal" app with those calculated by the registered dietitian based on a 3-day record of weighed/measured food (Table 1). For the intake of overall energy and each nutrient, including protein, lipid, carbohydrate, fiber, and salt, all ICCs were over 0.81 , indicating high reliability. Bland-Altman analysis, a method of evaluate the bias between two methods, demonstrated no significant bias between the "Calomeal" app and the estimates of the registered dietician $[14,15]$.

\section{Endpoints}

Compared with baseline data, statistically significant reductions were observed in HbAlc, glycated albumin (GA), BW, and BMI at 3 months after patients began using the app (Table 2). HbA1c decreased from $7.9(7.58-8.23) \%$ to $7.6(7.3$ - 8.23)\% $(\mathrm{P}=0.0410)$, and BMI decreased from 25.17 (21.63 - 28.59) to $24.54(21.57-27.81) \mathrm{kg} / \mathrm{m}^{2}(\mathrm{P}=0.0057)$. There were no statistically significant differences in other parameters between baseline and 3 months. The DTSQ score and PAID score improved non-significantly, while the intake of energy, lipid, and carbohydrate decreased non-significantly.

\section{Factors associated with the reduction of $\mathrm{HbA1c}$ and BMI}

We applied Spearman's rank correlation coefficient to identify 
Table 2. Data at Baseline and at the End of the Study (3 months After Baseline)

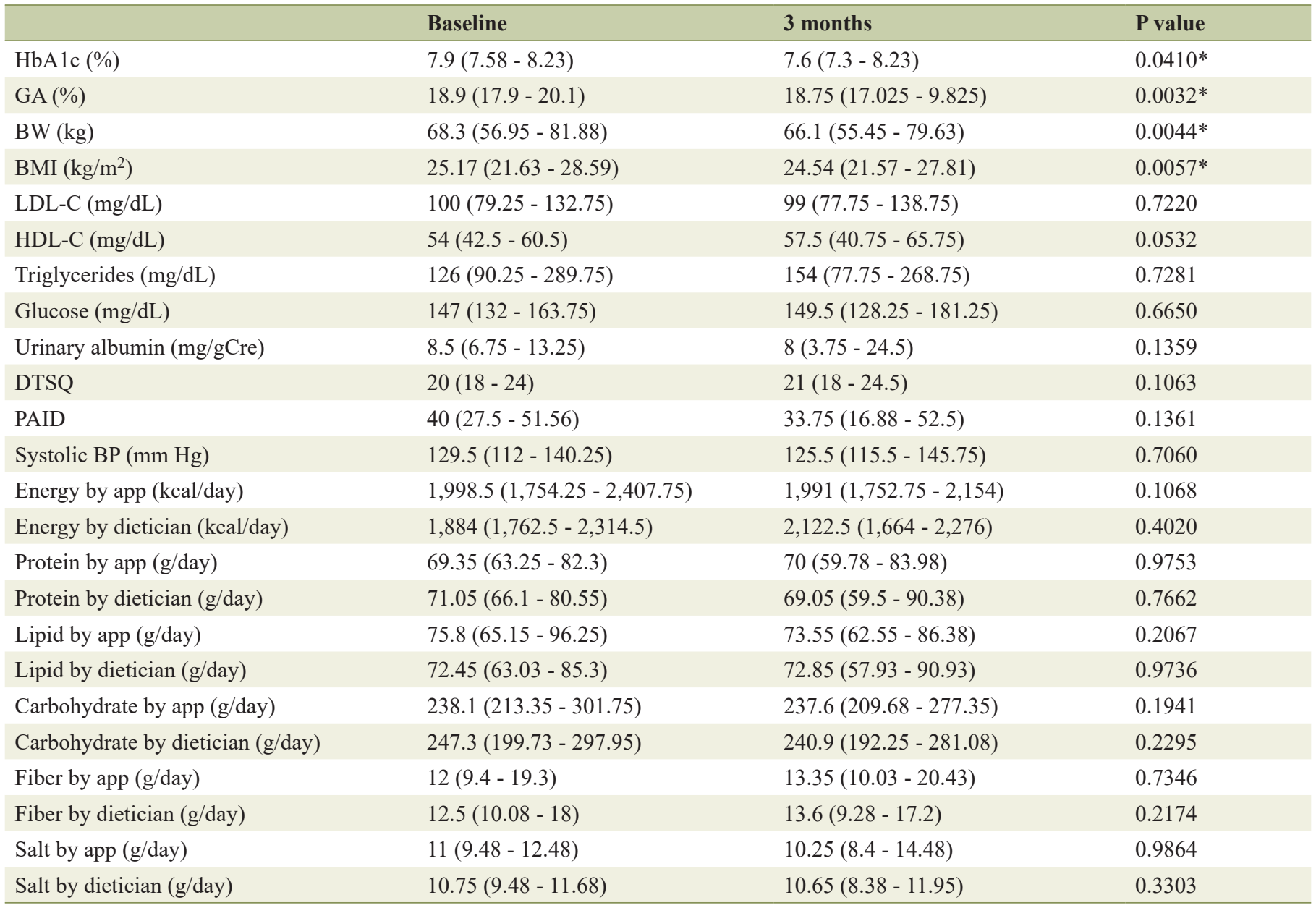

Total $n=18$. Data are expressed as means \pm SD for normally distributed data and medians (interquartile range) for data with skewed distributions. *A $P$ value < 0.05 denotes the presence of a statistically significant difference. HbA1c: hemoglobin A1c; GA: glycated albumin; BW: body weight; BMI: body mass index; LDL-C: low-density lipoprotein cholesterol; HDL-C: high-density lipoprotein cholesterol; DTSQ: diabetes treatment satisfaction questionnaire; PAID: problem areas in diabetes; BP: blood pressure; app: application.

factors associated with the reduction of $\mathrm{HbA1c}$ and BMI (Table 3). $\Delta \mathrm{HbA} 1 \mathrm{c}$ over 3 months correlated with $\Delta \mathrm{GA}(\mathrm{P}=0.0449)$ and with $\Delta$ carbohydrate as estimated by the app $(\mathrm{P}=0.0338) . \Delta \mathrm{BMI}$ over 3 months correlated with $\triangle \mathrm{BW}(\mathrm{P}<0.0001), \Delta$ energy $(\mathrm{P}$ $=0.0393)$, and $\Delta$ carbohydrate $(\mathrm{P}=0.0170)$ as estimated by the app (Fig. 2). These results suggested that a reduction of carbohydrate intake based on data provided by this app could be beneficial for improving glycemic and BW control.

\section{Adherence and the ability to estimate energy intake}

In this study, we defined adherence as logging meal records at least 3 days per week ( 2 weekdays and 1 weekend day). Over the course of 12 weeks, the mean percent adherence of the 18 patients was $80.3 \pm 27.3 \%$. Using the "Calomeal" app may improve the ability of patients to estimate their energy intake, and thus we evaluated these estimates at the beginning and end of the study. Patients wrote down their estimated energy intake for each meal on a 3-day food record. An estimate was defined as correct if it was within $10 \%$ of the actual value. By the end of the study, the ability to correctly estimate energy intake had improved in nine patients, worsened in five, and remained the same in four.

\section{Discussion}

This study showed that the intervention by visualizing meal nutrients with the app significantly improved HbAlc and BMI in Japanese patients with type 2 diabetes mellitus. An important point regarding the "Calomeal" app is that according to ICCs and Bland-Altman analysis, its nutrient estimates demonstrated reliability and validity compared to the estimates of the registered dietitian based on a 3-day record of weighed/measured food.

The intervention in this study involved the use of an app on a smartphone, an indispensable device in modern life, to visualize BW, target energy and nutrient intake, and intake over daily, 
Table 3. Correlations of $\triangle \mathrm{HbA} 1 \mathrm{c}$ and $\triangle \mathrm{BMI}$ With Other Clinical Parameters Over the 3-Month Study Period

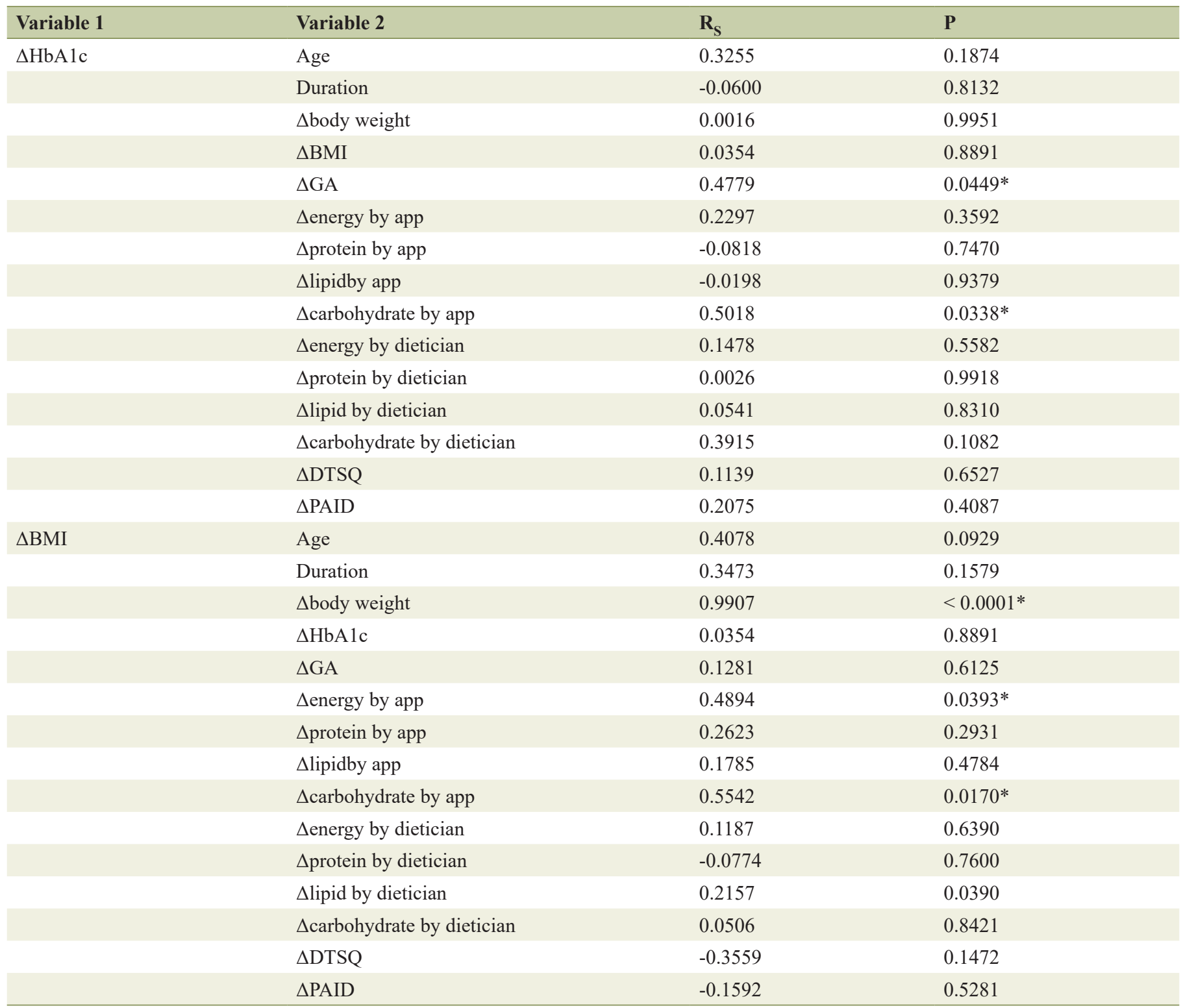

*A P value < 0.05 denotes the presence of a statistically significant difference. HbA1c: hemoglobin A1c; GA: glycated albumin; BMI: body mass index; DTSQ: diabetes treatment satisfaction questionnaire; PAID: problem areas in diabetes; app: application.

weekly, and monthly periods. Patients could determine what they should eat based on displayed data. For example, messages such as "overeating" or "need to ingest more protein" might help improve the self-management of diabetes mellitus.

Medical nutrition therapy with registered dieticians is known to effectively improve glycemic control, and it is recommended that patients start it in the early stages of type 2 diabetes mellitus and continue it throughout their lives [3-5, 16-18]. The term "medical nutrition therapy" was defined in 1994 by the American Diabetes Association as the use of specific nutrition services to treat an illness, with two phases: 1) assessment of the nutritional status of the patient; and 2) the provision of treatment that includes nutrition therapy, coun- seling, and the use of specialized nutrition supplements [19]. Our study showed that the healthcare app such as "Calomeal," which is effective in both of these phases partially, may be a beneficial clinical choice for medical nutrition therapy in Japanese patients. Merits of using smartphone apps for medical therapy include their convenience, however we must also consider individuals who are not proficient at using smartphones (for example, older patients), those with certain physical and mental disabilities, and those who live in remote areas with extreme socioeconomic deprivation [8].

While the intake of energy and carbohydrate did not decrease significantly as a whole, the reductions in HbA1c and BMI significantly correlated with the reduction in carbo- 

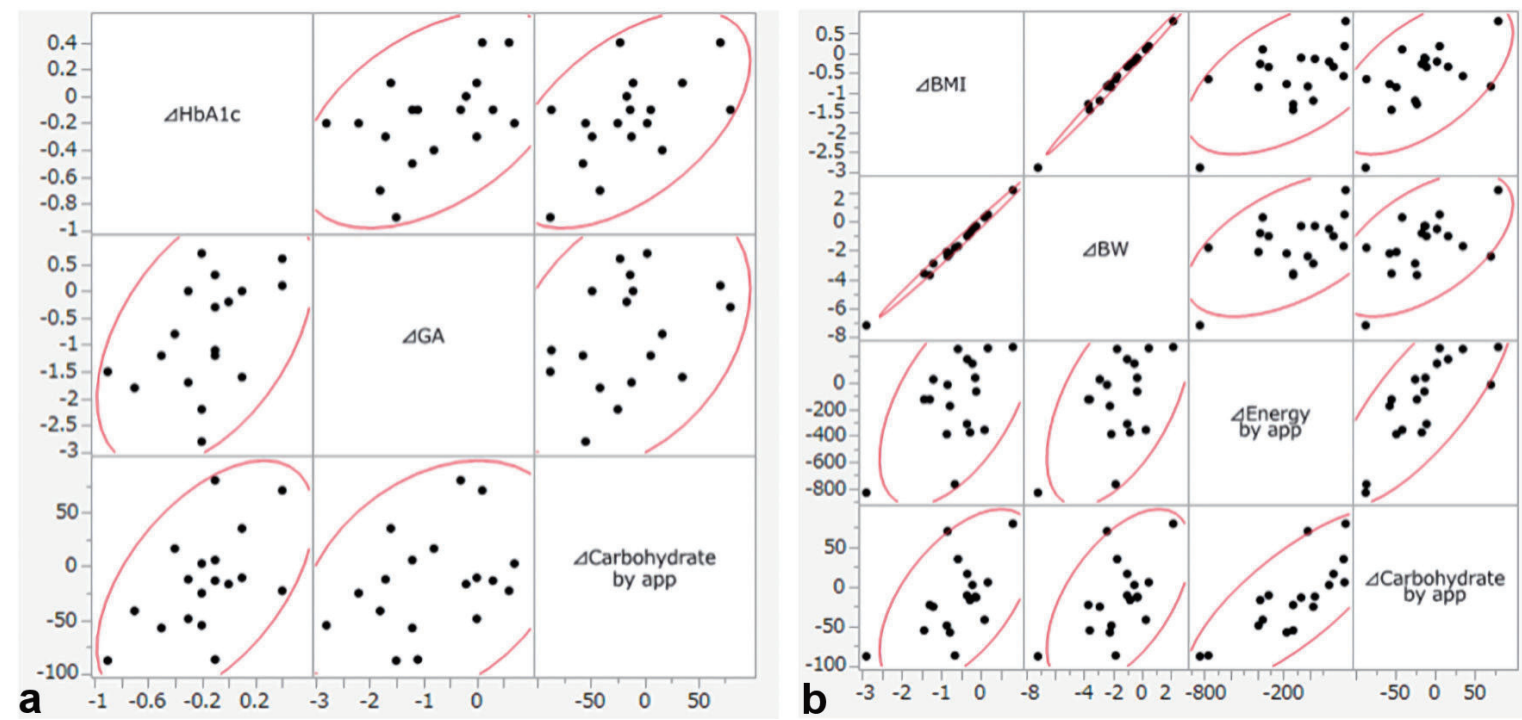

Figure 2. (a) Scatter plot of $\Delta \mathrm{HbA} 1 \mathrm{c}, \Delta \mathrm{GA}$ and $\Delta$ carbohydrate by app. (b) Scatter plot of $\Delta \mathrm{BMI}, \Delta \mathrm{BW}, \Delta$ energy and $\Delta$ carbohydrate by app. HbA1c: hemoglobin A1c; GA: glycated albumin; BMI: body mass index; BW: body weight; app: application.

hydrate intake as estimated by the app $(\mathrm{P}=0.0338$ and $\mathrm{P}=$ 0.0170 , respectively). The data suggest the possibilities that the app contributed to a reduction of carbohydrate intake, which may be one of the main reasons for the improvement in glycemic and BW control in some patients, as shown in previous studies [17, 20, 21].

On the other hand, QOL scores did not improve over the course of the study. In a post hoc evaluation, we divided the patients into two groups, one in which DTSQ scores improved, and the other in which they did not. The changes in carbohydrate intake in each group were not significantly different. However, there was a significant difference in deviation from the target carbohydrate intake $(50 \%$ energy) throughout the study period. The deviation from the target intake was significantly lower in the group whose DTSQ score improved than the group in which it did not $(\mathrm{P}=0.0319)$. These results suggested that patients' treatment satisfaction scores were higher when their carbohydrate intake was closer to the target $(50 \%$ energy). In another words, although our data suggest that the reduction of carbohydrate intake as a result of using the app was one of the main reasons for improvement in glycemic and BW control in some patients, an excessive decrease in carbohydrate intake below the target level may reduce QOL. In addition, according to a study with more than two decades of follow-up that investigated mortality risk in a large cohort of adults in the USA, mid-life dietary patterns characterized both by low-carbohydrate $(<40 \%$ of energy from carbohydrates) and high-carbohydrate ( $>70 \%$ of energy from carbohydrates) consumption were associated with increased mortality risk and shorter residual lifespan; the minimum risk was observed in individuals who obtained $50-55 \%$ of their energy from carbohydrates [22]. Accordingly, a moderate reduction in carbohydrate intake seems to be most appropriate for patients with diabetes mellitus.

Adherence of using the app and the ability to estimate energy intake of the patients did not show any significant cor- relations with the improvement of HbAlc and BMI. Over the course of 3 months, the mean percent adherence was $80.3 \pm 27.3 \%$, when it was required to log meal records only at least 3 days per week. It is not certain that the patients are able to use the app for more than 3 months in real life.

The additional issues that require attention are frailty and sarcopenia associated with diabetes mellitus and accelerated aging. Both quantitative (energy intake) and qualitative nutritional factors were shown to contribute to the development of frailty and sarcopenia in older adults [23, 24]. Considering these problems, the healthcare app "Calomeal" and its ability to display graphs of both actual and target daily intake may be helpful, if the accuracy and proper use of data are sometimes assessed by registered dieticians.

The findings of this study should be considered in the context of several limitations. First, energy and carbohydrate did not decrease significantly as a whole, although the reductions in $\mathrm{HbA} 1 \mathrm{c}$ and BMI significantly improved and correlated with the reduction in carbohydrate intake as estimated by the app. We have to consider and evaluate other factors too, besides meals, which improve HbA1c and BMI, for example, behavior change including strengthening exercises, stopping smoking, etc., about which we did not consider in this study. Also, the reductions in $\mathrm{HbA} 1 \mathrm{c}$ and BMI did not significantly correlated with the reduction in carbohydrate intake as estimated by the dietician, although the app's estimates demonstrated reliability and validity compared to the estimates of the registered dietician. Second, it is because that this was the pilot study, there was no control group. A randomized study including control group, with larger size and longer duration will be needed. Third, we have to consider about the possibilities of bias, in case that the participants, with the average age $53.4 \pm 7.8$ years old, may have been familiar with or interested in participating in studies involving app use. Mobile app might be difficult to handle with, rather inconvenient for the elderly patients and for those with certain disabilities. Fourth, in this study, the patients 
did not receive any advice about nutrition therapy from either staff members or the registered dietician because we tried to evaluate the efficacy of mobile app itself. In the real life, we need to consider the proper methods of using mobile app in the system of medical nutrition therapy with registered dieticians.

In summary, visualizing nutrient content with the mobile app could be good intervention to improve glycemic control and BMI in Japanese patients over a short period. This kind of app may be clinically beneficial for self-management of Japanese patients with type 2 diabetes mellitus.

\section{Acknowledgments}

Parts of this study were presented in abstract form (oral session) at the 80th Scientific Sessions of the American Diabetes Association, Chicago, IL, June 12 - 16, 2020.

\section{Financial Disclosure}

This research received no specific grant from any funding agency in the public, commercial, or not-for-profit sectors.

\section{Conflict of Interest}

The authors have no conflict of interest to disclose.

\section{Informed Consent}

Each participant provided written informed consent.

\section{Author Contributions}

Substantial contributions to the conception or design of the work, or acquisition, analysis or interpretation of data: AT and JS. Drafting the work or revising it critically for important intellectual content: AT, JS and HW. Final approval of the version to be published: JS and HW. Agreement to be accountable for all aspects of the work and ensuring that questions related to the accuracy or integrity of any part of the work are appropriately investigated and resolved: AT, JS, SS, YI, ME, YS, MK, EM, YW, TF, TM,TU, TM, KA, TS, AK, HS, and HW.

\section{Data Availability}

The authors declare that the data supporting the findings of this study are available within the article.

\section{References}

1. National Institutes of Biomedical Innovation HaN, Nutrition NIoHa: The National Health and Nutrition Survey
(NHNS). Japan. 2016. Summary. https://www.nibiohn. go.jp/eiken/kenkounippon21/download_files/eiyouchousa/2016.pdf. Accessed Sep 222020.

2. Nanditha A, Ma RC, Ramachandran A, Snehalatha C, Chan JC, Chia KS, Shaw JE, et al. Diabetes in Asia and the Pacific: implications for the global epidemic. Diabetes Care. 2016;39(3):472-485.

3. Davies MJ, D'Alessio DA, Fradkin J, Kernan WN, Mathieu C, Mingrone G, Rossing P, et al. Management of hyperglycemia in type 2 diabetes, 2018. A consensus report by the American Diabetes Association (ADA) and the European Association for the Study of Diabetes (EASD). Diabetes Care. 2018;41(12):2669-2701.

4. American Diabetes Association. 5. Facilitating behavior change and well-being to improve health outcomes: standards of medical care in diabetes-2020. Diabetes Care. 2020;43(Suppl 1):S48-S65.

5. Yamauchi $\mathrm{T}$, Kamiya $\mathrm{H}$, Utsunomiya $\mathrm{K}$, Watada $\mathrm{H}$, Kawanami D, Sato J, Kitada M, et al. Medical nutrition therapy and dietary counseling for patients with diabetesenergy, carbohydrates, protein intake and dietary counseling. Diabetol Int. 2020;11(3):224-239.

6. Drincic A, Prahalad P, Greenwood D, Klonoff DC. Evidence-based Mobile Medical Applications in Diabetes. Endocrinol Metab Clin North Am. 2016;45(4):943-965.

7. Veazie S, Winchell K, Gilbert J, Paynter R, Ivlev I, Eden K, Nussbaum K, et al.: In: Mobile Applications for SelfManagement of Diabetes. Rockville (MD), 2018.

8. Fleming GA, Petrie JR, Bergenstal RM, Holl RW, Peters AL, Heinemann L. Diabetes digital app technology: benefits, challenges, and recommendations. A consensus report by the European Association for the Study of Diabetes (EASD) and the American Diabetes Association (ADA) diabetes technology working group. Diabetes Care. 2020;43(1):250-260.

9. Haneda M, Noda M, Origasa H, Noto H, Yabe D, Fujita Y, Goto A, et al. Japanese Clinical Practice Guideline for Diabetes 2016. Diabetol Int. 2018;9(1):1-45.

10. Bradley C. Diabetes treatment satisfaction questionnaire (DTSQ), Handbook of psychology and diabetes: a guide to psychological measurement in diabetic research and practice. Chur, Switzerland: Harwood Academic Publishers; 1994.

11. Dennick K, Sturt J, Speight J. What is diabetes distress and how can we measure it? A narrative review and conceptual model. J Diabetes Complications. 2017;31(5):898-911.

12. Polonsky WH, Anderson BJ, Lohrer PA, Welch G, Jacobson AM, Aponte JE, Schwartz CE. Assessment of diabetes-related distress. Diabetes Care. 1995;18(6):754-760.

13. McHugh ML. Interrater reliability: the kappa statistic. Biochem Med (Zagreb). 2012;22(3):276-282.

14. Bland JM, Altman DG. Statistical methods for assessing agreement between two methods of clinical measurement. Lancet. 1986;1(8476):307-310.

15. Giavarina D. Understanding Bland Altman analysis. Biochem Med (Zagreb). 2015;25(2):141-151.

16. Pastors JG, Warshaw H, Daly A, Franz M, Kulkarni $\mathrm{K}$. The evidence for the effectiveness of medical nutrition therapy in diabetes management. Diabetes Care. 
2002;25(3):608-613.

17. Huang MC, Hsu CC, Wang HS, Shin SJ. Prospective randomized controlled trial to evaluate effectiveness of registered dietitian-led diabetes management on glycemic and diet control in a primary care setting in Taiwan. Diabetes Care. 2010;33(2):233-239.

18. Moller G, Andersen HK, Snorgaard O. A systematic review and meta-analysis of nutrition therapy compared with dietary advice in patients with type 2 diabetes. Am J Clin Nutr. 2017;106(6):1394-1400.

19. Identifying patients at risk: ADA's definitions for nutrition screening and nutrition assessment. Council on Practice (COP) Quality Management Committee. J Am Diet Assoc. 1994;94(8):838-839.

20. Sato J, Kanazawa A, Makita S, Hatae C, Komiya K, Shimizu T, Ikeda F, et al. A randomized controlled trial of $130 \mathrm{~g} /$ day low-carbohydrate diet in type 2 diabetes with poor glycemic control. Clin Nutr. 2017;36(4):992-1000.
21. Huntriss R, Campbell M, Bedwell C. The interpretation and effect of a low-carbohydrate diet in the management of type 2 diabetes: a systematic review and metaanalysis of randomised controlled trials. Eur J Clin Nutr. 2018;72(3):311-325.

22. Seidelmann SB, Claggett B, Cheng S, Henglin M, Shah A, Steffen LM, Folsom AR, et al. Dietary carbohydrate intake and mortality: a prospective cohort study and meta-analysis. Lancet Public Health. 2018;3(9):e419-e428.

23. Park SW, Goodpaster BH, Strotmeyer ES, Kuller LH, Broudeau R, Kammerer C, de Rekeneire N, et al. Accelerated loss of skeletal muscle strength in older adults with type 2 diabetes: the health, aging, and body composition study. Diabetes Care. 2007;30(6):1507-1512.

24. Sinclair AJ, Abdelhafiz AH, Rodriguez-Manas L. Frailty and sarcopenia - newly emerging and high impact complications of diabetes. J Diabetes Complications. 2017;31(9):1465-1473. 